\title{
GABAergic projections to the oculomotor nucleus in the goldfish (Carassius auratus)
}

\author{
M. Angeles Luque, Julio Torres-Torrelo, Livia Carrascal, Blas Torres, Luis Herrero* \\ Department of Physiology and Zoology, University of Seville, Seville, Spain
}

\section{Edited by:}

Fernando Martinez-Garcia, Universidad de Valencia, Spain

Reviewed by:

Hans Straka, Ludwig Maximilian

University Munich, Germany

Julian Yañez, University of A Coruña,

Spain

\section{*Correspondence:}

Luis Herrero, Departamento de

Fisiología y Zoología, Facultad de

Biología, Avenida Reina Mercedes 6,

41012 Sevilla, Spain

e-mail:Iherrero@us.es
The mammalian oculomotor nucleus receives a strong $\gamma$-aminobutyric acid (GABA)ergic synaptic input, whereas such projections have rarely been reported in fish. In order to determine whether this synaptic organization is preserved across vertebrates, we investigated the GABAergic projections to the oculomotor nucleus in the goldfish by combining retrograde transport of biotin dextran amine, injected into the antidromically identified oculomotor nucleus, and GABA immunohistochemistry. The main source of GABAergic afferents to the oculomotor nucleus was the ipsilateral anterior octaval nucleus, with only a few, if any, GABAergic neurons being located in the contralateral tangential and descending nuclei of the octaval column. In mammals there is a nearly exclusive ipsilateral projection from vestibular neurons to the oculomotor nucleus via GABAergic inhibitory inputs; thus, the vestibulooculomotor GABAergic circuitry follows a plan that appears to be shared throughout the vertebrate phylogeny. The second major source of GABAergic projections was the rhombencephalic reticular formation, primarily from the medial area but, to a lesser extent, from the inferior area. A few GABAergic oculomotor projecting neurons were also observed in the ipsilateral nucleus of the medial longitudinal fasciculus. The GABAergic projections from neurons located in both the reticular formation surrounding the abducens nucleus and the nucleus of the medial reticular formation have primarily been related to the control of saccadic eye movements. Finally, all retrogradely labeled internuclear neurons of the abducens nucleus, and neurons in the cerebellum (close to the caudal lobe), were negative for GABA. These data suggest that the vestibuloocular and saccadic inhibitory GABAergic systems appear early in vertebrate phylogeny to modulate the firing properties of the oculomotor nucleus motoneurons.

Keywords: oculomotor system, vestibuloocular reflex, saccadic eye movements, GABA immunohistochemistry, fish

\section{INTRODUCTION}

Based on immunohistochemical studies, the mammalian oculomotor nucleus has been reported to receive a strong $\gamma$-aminobutyric acid (GABA)ergic synaptic input (de la Cruz et al., 1992; Spencer et al., 1992; Wentzel et al., 1996). Electrophysiological investigations have demonstrated an ipsilateral disynaptic inhibition from the labyrinth to the oculomotor nucleus motoneurons; this inhibitory postsynaptic potential is blocked by the GABA antagonists bicuculline and picrotoxin (Ito et al., 1970; Highstein, 1973; Precht et al., 1973; Uchino and Suzuki, 1983). These data, together with those showing a high density of GABA-immunoreactive vestibular neurons (Highstein and Holstein, 2006), suggest that this neurotransmitter plays a key role in mediating the vertical vestibuloocular reflex (Highstein and McCrea, 1988). It has also been proposed that GABA is utilized as an inhibitory neurotransmitter by premotor neurons related to the control of horizontal and vertical saccadic eye movements (de la Cruz et al., 1992; Spencer and Wang, 1996; Horn et al., 2003). The oculomotor system in the goldfish provides a suitable model for comparative research as these animals exhibit compensatory eye movements in response to head and visual-field displacements, together with a marked pattern of spontaneous saccades in the horizontal plane (Easter, 1971; Pastor et al., 1992; Keng and Anastasio, 1997; Mensh et al., 2004). The motoneurons innervating the eye muscles in the goldfish lie in similar locations to those found in other vertebrates (Graf and McGurk, 1985), and their firing properties resemble those reported in both cats and monkeys (Delgado-Garcia et al., 1986a; Fuchs et al., 1988; de la Cruz et al., 1989). Furthermore, the extraocular motor nuclei in the goldfish show GABAergic innervation, and the stimulation of the vestibular nerve produces ipsilateral inhibitory postsynaptic potentials (Graf et al., 1997). Finally, the structures projecting to the ocular motor nuclei in the goldfish are similar to those reported in mammals (Allum et al., 1981; Torres et al., 1992, 1995). Based on these similarities, the present study was designed to investigate the GABAergic projections to the oculomotor nucleus in the goldfish, in order to determine if this inhibitory circuitry is preserved throughout vertebrate phylogeny.

\section{MATERIALS AND METHODS}

Experiments were carried out in 10-15 cm long goldfish (Carassius auratus), which were obtained from local suppliers. All studies were performed in accordance with the European Community Directive 2003/65, as well as with the Spanish Royal Decree 120/2005 and University of Seville regulations on the care of laboratory animals. Under general anesthesia (tricaine methanesulfonate, MS222, 1:2000 wt/vol, Sigma-Aldrich), the animal was clamped between two sponge rubber pads inside a home-made Perspex water chamber. The mouth was fitted to a plastic tube connected to a 
well aerated recirculating system propelled by a pump to ensure a constant flow over the gills. The cranial bones and the tissues overlying the tectal and telencephalic lobes were gently removed to avoid bleeding, after which an insulated silver $(250 \mu \mathrm{m}$ diameter) bipolar electrode was implanted in the left oculomotor nerve with a micromanipulator (Figure 1A). The final location of the stimulating electrode was such that a single short pulse $(50 \mu \mathrm{s})$ of low current strength $(<100 \mu \mathrm{A})$ evoked a twitch of the medial rectus muscle, leading to a nasally directed eye movement. The left oculomotor nucleus was identified by recording the antidromic field potential (Figure 1B) with a $2-\mathrm{M} \mathrm{NaCl}$-filled glass micropipette (1-5 M $\Omega$ ). This microelectrode was then exchanged for a glass micropipette (10-15 $\mu \mathrm{m}$ tip diameter) filled with $10 \%$ biotin dextran amine (BDA, Invitrogen) in phosphate buffered saline (PBS, $0.1 \mathrm{M}, \mathrm{pH}=7.4$ ). The micropipette was driven to the oculomotor nucleus, which was again identified based on the antidromic field potential. Cathodal pulses (10 s on followed by $5 \mathrm{~s}$ off) of 5-7 $\mu \mathrm{A}$ were delivered over a period of 15-20 min. After injection, the piece of bone removed during surgery was restored and fixed in place.

Following 7-10 days of postoperative survival time, the fish was again anesthetized in MS222, then transcardially perfused with $100 \mathrm{ml}$ teleost Ringer's solution, followed by $200 \mathrm{ml}$ of a fixative solution (4\% paraformaldehyde, $0.08 \%$ glutaraldehyde, and 15\% picric acid in $0.1 \mathrm{M}$ phosphate buffer, $\mathrm{pH}=7.4$ ). The brain was removed from the skull and cut serially in a cryostat into $50 \mu \mathrm{m}$ transverse sections. Free-floating sections were washed in PBS and incubated for $1 \mathrm{~h}$ at room temperature in $10 \%$ normal goat serum (Sigma-Aldrich). They were then exposed to rabbit anti-GABA (1:1000, Sigma-Aldrich) overnight at room temperature and to goat anti-rabbit IgG-Cy3 (1:400, Jackson Immunoresearch) for $2 \mathrm{~h}$. To visualize the BDA tracer, the sections were treated with $2 \%$ fetal bovine serum for $1 \mathrm{~h}$, before being incubated with antibiotin-FITC (1:120, Sigma-Aldrich) for $2 \mathrm{~h}$. To reveal the location of the injection site in relation to the oculomotor nucleus, the motoneurons were immunohistochemically stained for choline acetyl transferase (ChAT). Transverse mesencephalic sections were incubated with $3 \%$ rabbit serum for $1 \mathrm{~h}$, followed by a solution of goat anti-ChAT antibody (1:100, Millipore) overnight at room temperature, and rabbit anti-goat IgG-Cy3 (1:200, Jackson ImmunoResearch Labs.) for $2 \mathrm{~h}$. All solutions were prepared in a PBS solution containing $0.3 \%$ Triton X-100. Furthermore, the specificity of the immunolabeling was tested by incubating some sections without the primary antibody. Additional information about the specificity of the primary antibody used in the present study was provided by Sigma; that is, rabbit anti-GABA shows positive binding with GABA, in a dot blot assay, and negative binding with BSA. Finally, the sections were placed on gelatinized glass slides and coverslipped with fluorescent mounting medium (Dako Cytomation). Sections were first analyzed using a fluorescence microscope (Olympus BX61), then photographed in a confocal microscope (Leica TCS-SP2). Data were collected from six experiments in which the central core of the injection site lay in the oculomotor nucleus, the BDA retrograde transport was deemed to be effective because stained cells were located as distant as the inferior rhombencephalic reticular formation, and GABA showed good penetration ( $>60 \%$ of the section thickness). Both retrogradely labeled and GABA-positive cells were scored (stated as mean \pm SE) and mapped onto transverse schematics. Ipsilateral and contralateral neurons were depicted relative to the injection side. The average somal diameter (long axis + short axis/2) was measured. The nomenclature adopted in this study was that used by McCormick and Bradford (1994) and Meek and Nieuwenhuys (1998).

\section{RESULTS}

The location of the injection site within the oculomotor nucleus was first determined by the antidromic field potential evoked following the electrical microstimulation of the third nerve (Figure 1A). This field potential was characterized by a prominent negative voltage wave with a latency of less than $1 \mathrm{~ms}$ from the stimulus onset to the negative peak. The amplitude of the negative peak increased toward the center of the oculomotor nucleus (Figure 1B). In all cases $(n=6)$, the antidromic potential was recorded along 300$600 \mu \mathrm{m}$ in the dorso-ventral axis and exhibited a negative peak larger than $0.4 \mathrm{mV}$. The injection site was also characterized by the extent of the central core and spread area of the BDA tracer in relation to the labeling of the motoneurons (revealed by ChAT immunohistochemistry). The central core of the injection site was confined to the oculomotor cellular column close to the midline (Figures 1C,D), extending from the rostral to the caudal pole of the nucleus $(-500 \mu \mathrm{m})$. In other words, the region of effective BDA uptake included all motoneuronal pools (that is, the motoneurons innervating the ipsilateral medial rectus, the inferior rectus, and the inferior oblique eye muscles) of the oculomotor nucleus in the goldfish (Graf and McGurk, 1985). In the contralateral (noninjected) oculomotor nucleus, some cell bodies positive for both ChAT and BDA were observed (see arrow in Figure 1D); these probably correspond to the motoneurons innervating the contralateral superior rectus and/or superior oblique eye muscles. However, the vast majority of the cells in the non-injected nucleus (see arrowheads in Figure 1D) were only positive for ChAT, even though they were located close to the midline. The spread area of the injection site reached the dorso-lateral aspects of the oculomotor nucleus (where some of the motoneurons innervating the ipsilateral inferior rectus and inferior oblique eye muscles are located), the ipsilateral medial longitudinal fasciculus and, to a lesser extent, the contralateral oculomotor nucleus.

Retrogradely labeled GABA-positive neurons were more numerous in the octaval column nuclei, less abundant within the rhombencephalic reticular formation, and very scarce within the nucleus of the medial longitudinal fasciculus (Figure 2). Within the nuclei of the octaval column, GABAergic neurons projecting to the oculomotor nucleus were located almost exclusively in the ipsilateral anterior octaval nucleus, and only occasionally in the contralateral tangential and descending nuclei. A group of labeled neurons $(31.7 \pm 3.4)$ was found in the ipsilateral anterior octaval nucleus and, of these cells, a high proportion were also positive for GABA ( $45 \%$; Figures $2 \mathrm{C}, \mathrm{D}$ and $3 \mathrm{~A})$. Single- and doublelabeled cells, the somata of which were spherical or multipolar $(19.6 \pm 3.4 \mu \mathrm{m})$, were arranged in a rounded cluster, located in the cerebellar peduncle rostral to the entry of the eighth nerve. The axons of these cells traveled toward the ipsilateral medial longitudinal fasciculus, and in some cases were also positive for GABA (Figure 3B). In addition, a few stained neurons were located in 


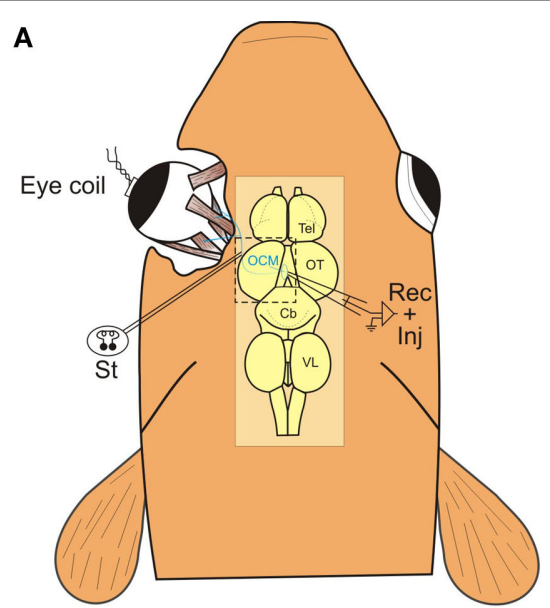

C

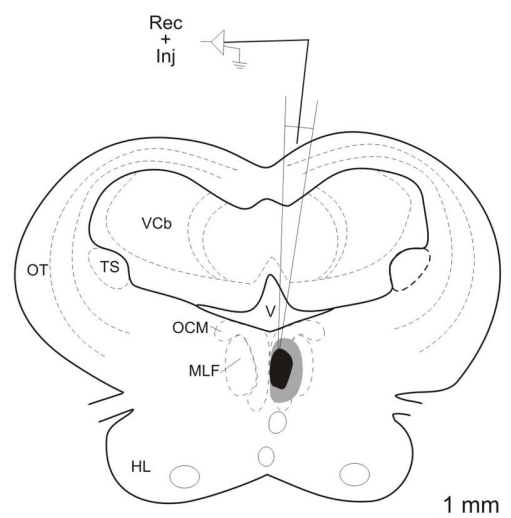

FIGURE 1 | Electrophysiological identification of the oculomotor nucleus and location of the injection site. (A) Schematic representation of the experimental design for the antidromic identification of the oculomotor nucleus following electrical stimulation (St) of the third cranial nerve. The location of the oculomotor nucleus and the trajectory of the third nerve are schematically illustrated in blue. (B) Antidromic field potentials recorded in the oculomotor nucleus following oculomotor nerve stimulation (St; triangle shows the onset of the stimulus). Recordings were obtained at several depths (indicated in $\mathrm{mm}$ ) as the electrode

\section{B}

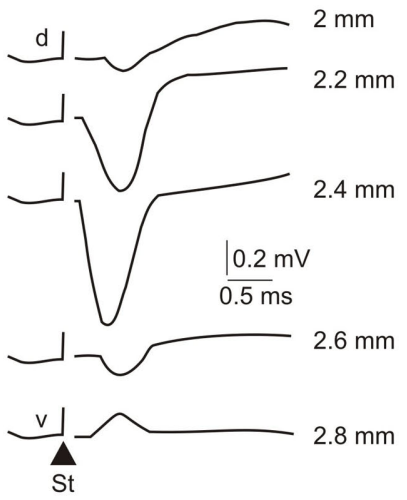

D

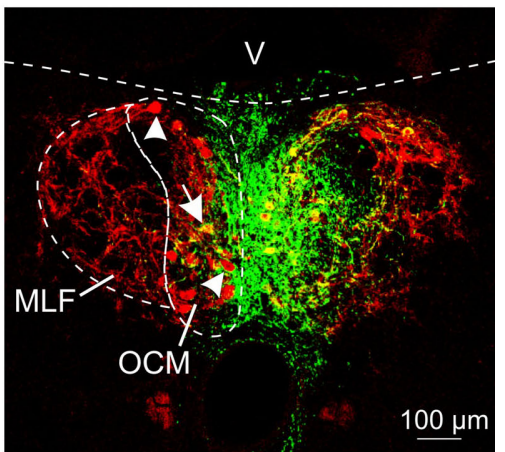

was driven from the dorsal (d) to the ventral (v) part of the nucleus. (C) Schematic drawing showing the location of the BDA injection site at the level of the oculomotor nucleus; the central core is shown in black and the spread area of the injection site in gray. (D) Confocal photomicrograph of the injection site. The green area corresponds to the deposit of BDA and the motoneurons are stained in red after immunohistochemistry against ChAT. Some ChAT-positive motoneurons (arrowheads) and a double-labeled motoneuron (arrow) can be seen contralateral to the injected oculomotor nucleus. Anatomical abbreviations in Figure 2. the contralateral anterior octaval nucleus $(6.6 \pm 1.9)$ and in the contralateral magnocellular nucleus $(5.1 \pm 3.1)$, but none of these was positive for GABA (Figures 2C,D). Caudal to the anterior octaval nucleus, labeled neurons were observed in the contralateral tangential $(31.4 \pm 7.3)$ and descending $(33.5 \pm 5.7)$ octaval nuclei. Although a few ( $<5$ per experiment) of the cells in these two nuclei were also positive for GABA, such double-labeled cells were only observed in two of the six cases analyzed. The somata of these double-labeled neurons were spherical $(18.6 \pm 1.7 \mu \mathrm{m})$ and fusiform $(18.1 \pm 2.1 \mu \mathrm{m})$, respectively, in the tangential and descending nuclei.

Retrogradely labeled neurons, some of which were positive for GABA $(<25 \%$, for each side), were also found in the rhombencephalic reticular formation (Figures $2 \mathrm{E}-\mathrm{I}$ ), distributed bilaterally in relation to the injection site, but with contralateral predominance. These cells, which were mainly multipolar $(21.5 \pm 3.4 \mu \mathrm{m}$; Figures $3 \mathrm{C}, \mathbf{D})$, were located throughout the medial reticular area (contralateral, $25.2 \pm 6.7$; ipsilateral, $15.3 \pm 5.8$ ), and in the rostral pole of the inferior reticular area (contralateral, $12.8 \pm 3.2$; ipsilateral, $5.1 \pm 1.9$ ). Occasionally (three of six experiments), neurons were also observed in the caudal pole of the superior reticular area. In addition, neurons projecting to the oculomotor nucleus were bilaterally placed in the nucleus of the medial longitudinal fasciculus or close to it (ipsilateral, $7.5 \pm 3.2$; contralateral $3.3 \pm 3.2$ ); a few of these cells ( $<2$ per experiment), lying ipsilateral to the injection site, were also positive for GABA. Finally, GABA-negative neurons (putative eurydendroid cells) were found just above the granular cell layer of the cerebellar vestibulolateral lobe $(23.2 \pm 4.1$; Figures 2C,D) and close to the contralateral caudal abducens nucleus (putative abducens internuclear neurons). These latter neurons were distributed in two separated clusters: one close to the caudal abducens nucleus (18.1 \pm 5.1 , Figure 2F) and the other one posterior to it (17.2 \pm 6.7 , not illustrated). 


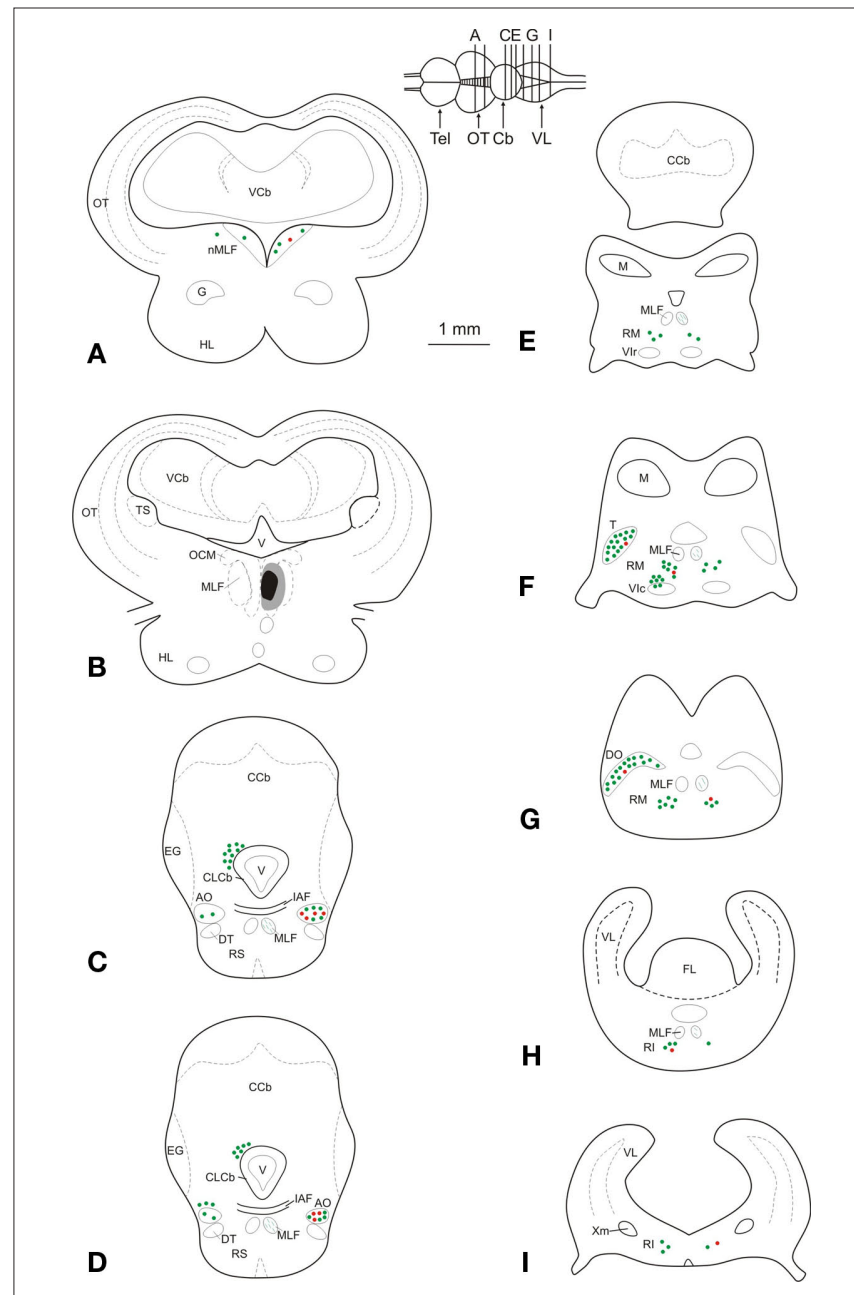

FIGURE 2 | (A-I) Schematic maps of transverse sections through the mesencephalon and the rhombencephalon in a representative experiment, illustrating the injection site, as well as the location of retrogradely labeled GABA-immunonegative (green dots) and GABA-immunoreactive (red dots) neurons. Each dot corresponds to two labeled neurons. Abbreviations: $\mathrm{AO}$, anterior octaval nucleus; $\mathrm{Cb}$, cerebellum; $\mathrm{CCb}$, corpus cerebelli; $\mathrm{CLCb}$, caudal lobe of the cerebellum; $\mathrm{DO}$, descending octaval nucleus; $\mathrm{DT}$, descending trigeminal tract; $\mathrm{EG}$, eminentia granularis; $\mathrm{FL}$, facial lobe; $\mathrm{G}$, nucleus glomerulosus; $\mathrm{HL}$, hypothalamic lobe; IAF, internal arcuate fibers; $M$, nucleus medialis; MLF, medial longitudinal fasciculus; $n M L F$, nucleus of the medial longitudinal fasciculus; OCM, oculomotor nucleus; OT, optic tectum; Rl, inferior rhombencephalic reticular formation; RM, medial rhombencephalic reticular formation; $\mathrm{RS}$, superior rhombencephalic reticular formation; T, tangential nucleus; Tel, telencephalon; TS, torus semicircularis; $\mathrm{V}$, ventricle; $\mathrm{VCb}$, cerebellar valvula; $\mathrm{VIc}$, caudal subdivision of the abducens nucleus; Vlr, rostral subdivision of the abducens nucleus; $\mathrm{VL}$, vagal lobe; $\mathrm{Xm}$, vagal motor nucleus.

\section{DISCUSSION}

The present study demonstrates for the first time that the teleost oculomotor nucleus receives GABAergic projections from neurons lying in the nuclei of the octaval column, in the rhombencephalic reticular formation, and in the nucleus of the medial longitudinal fasciculus. In contrast, all of the retrogradely labeled neurons that we observed in the contralateral abducens nucleus and the vestibulolateral lobe of the cerebellum were
GABA negative. These results are discussed in the context of available information in mammals and interpreted in a functional context.

In mammals, the abducens nucleus is formed by motoneurons and internuclear neurons which project to the ipsilateral lateral rectus eye muscle and to the contralateral medial rectus population of the oculomotor nucleus, respectively. The abducens nucleus is physiologically involved in the generation of conjugate horizontal eye movements, which are produced by the contraction of the lateral rectus muscle in one eye and the medial rectus muscle in the other eye. The burst-tonic activity in abducens motoneurons drives the contraction of the lateral rectus muscle, while the abducens internuclear neurons provide a similar signal to the contralateral medial rectus population of the oculomotor nucleus, which innervates the medial rectus muscle (Delgado-Garcia et al., 1986a,b; Fuchs et al., 1988). The abducens internuclear synaptic endings labeled by anterograde transport are immunoreactive for glutamate and/or aspartate, which is consistent with the physiological and morphological features of the excitatory inputs to the medial rectus motoneurons (Nguyen and Spencer, 1999). The abducens internuclear neurons have already been proposed in the goldfish by retrograde labeling studies (Cabrera et al., 1992). This proposal is supported by the present findings which demonstrate that the abducens internuclear neurons in goldfish are not inhibitory, at least not via GABAergic input to the oculomotor nucleus. The vestibulolateral lobe, consisting of the eminentia granularis and the caudal lobe, is one of the cerebellar subdivisions in teleosts. Various findings have implicated the vestibulateral lobe in the teleost oculomotor system network: electrical microstimulation of this region elicits ipsiversive horizontal eye movements, and its Purkinje cells exhibit eye-velocity-related activity (Pastor et al., 1997). Furthermore, the eminentia granularis receives mechanoor vestibulo-sensitive inputs from numerous hindbrain sources, and Purkinje cells directly project from the caudal lobe to the vestibular nuclei (Straka et al., 2006). Another source of direct cerebellar output is provided by the eurydendroid cells (Finger, 1978; Ikenaga et al., 2005), which are equivalent to mammalian deep cerebellar nucleus neurons. Eurydendroid cells could underlie the efferent output from the cerebellar vestibulolateral lobe to the oculomotor nucleus, given the similarity of their somato-dendritic morphology (Torres et al., 1992) and their putative non-inhibitory character (as shown here, labeled cells were negative for GABA). Furthermore, this pathway seems to be particular to fish, as it has not been reported in mammals (Torres et al., 1992).

In mammals, the rostral interstitial nucleus of the medial longitudinal fasciculus and the interstitial nucleus of Cajal are located rostral to the oculomotor nucleus. These two nuclei, which contain premotor neurons that are related to vertical upward, downward, and torsional saccadic eye movements, project to the oculomotor nucleus, predominantly on the ipsilateral side (reviewed in Horn, 2006). Anatomical studies have revealed GABAergic neurons in the rostral interstitial nucleus of the medial longitudinal fasciculus and interstitial nucleus of Cajal projecting to the oculomotor nucleus, and these neurons could contribute to the control of vertical eye movements (Spencer and Wang, 1996; Horn et al., 2003). Studies of the region rostral to the oculomotor nucleus in the goldfish, which includes the nucleus of the medial longitudinal fasciculus, 
A

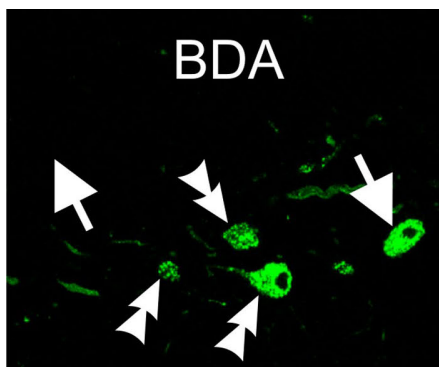

$50 \mu \mathrm{m}$

$\mathrm{AO}$

B

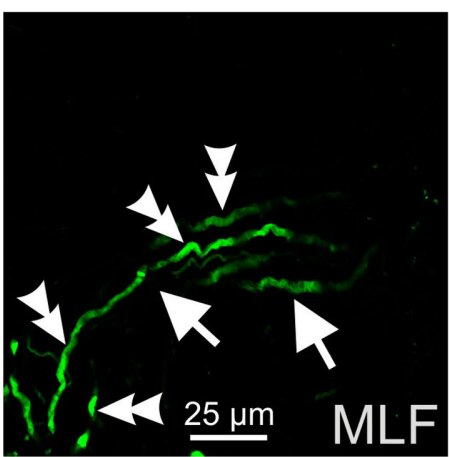

C

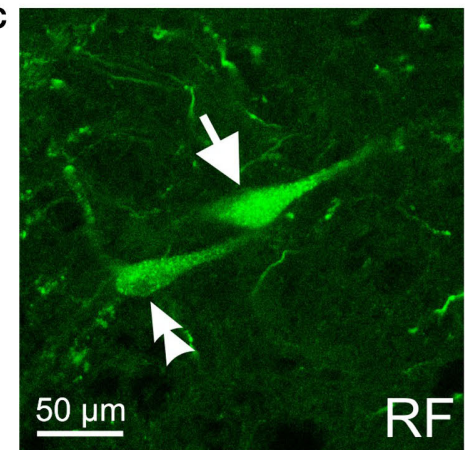

D

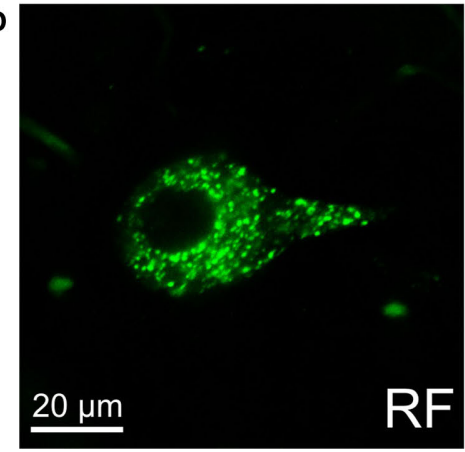

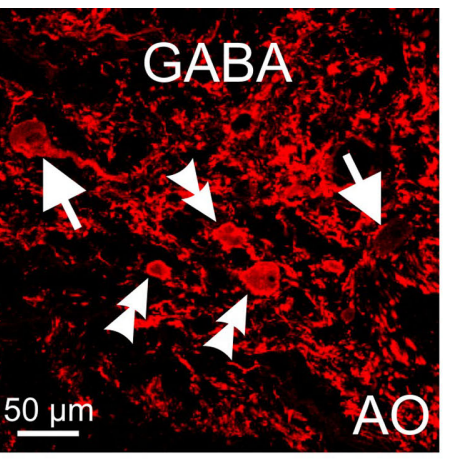
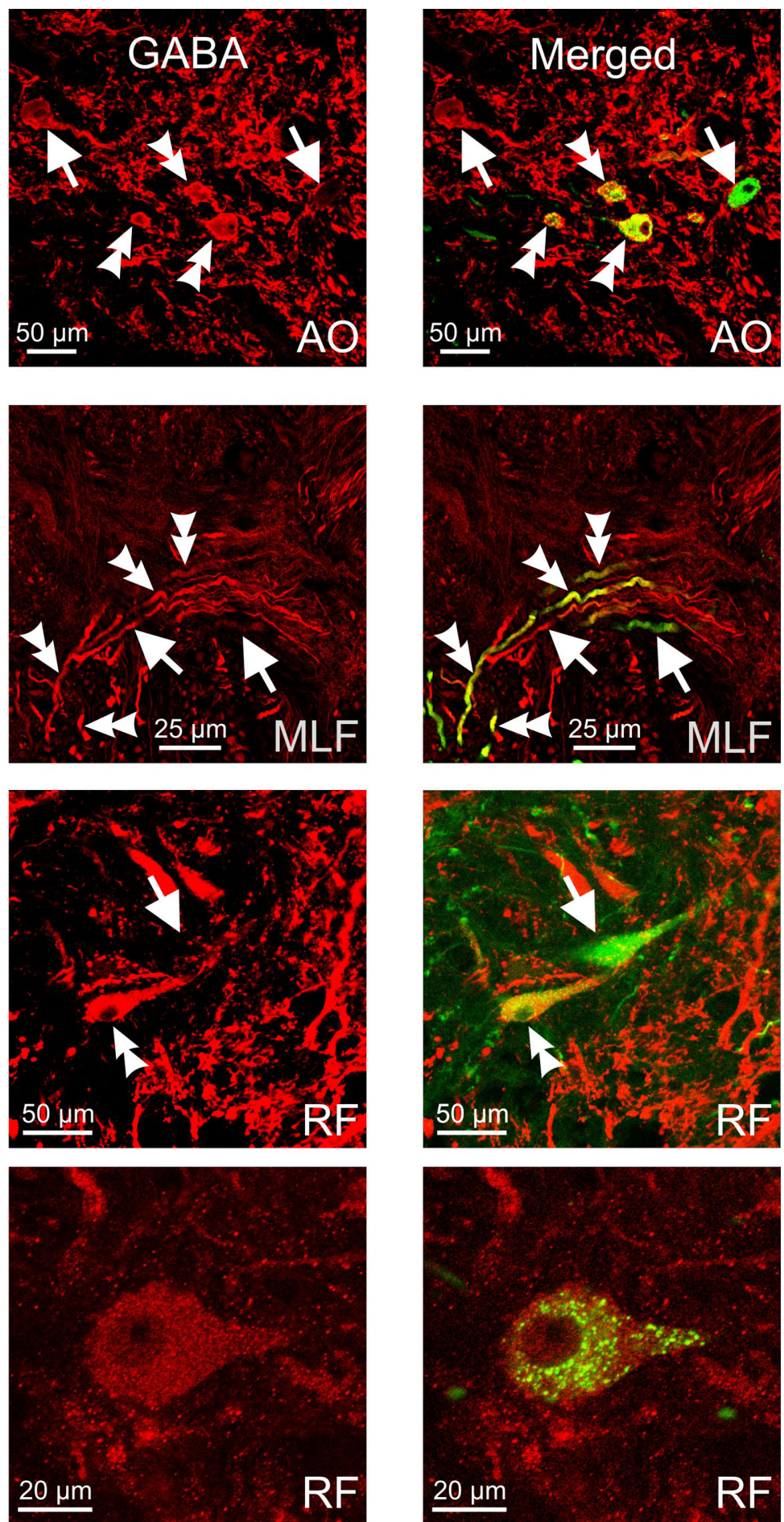

FIGURE 3 | Confocal photomicrographs of retrogradely labeled (green), GABA-immunoreactive (red) and double-labeled (yellow) neurons and fibers. (A) Neurons in the anterior octaval nucleus (AO). (B)

Axons running from the anterior octaval nucleus to the medial longitudinal fasciculus (MLF). (C,D) Neurons in the rhombencephalic reticular formation (RF). Double arrowheads indicate double-labeled cells and fibers; arrows indicate labeled cells/fibers positive for retrograde tracer or for GABA. have suggested that it is involved in locomotion (Kobayashi et al., 2009) but could also contribute to the encoding of vertical and horizontal saccadic eye movements (Luque et al., 2006). Neurons located within the nucleus of the medial longitudinal fasciculus or close to it were bilaterally labeled, with ipsilateral predominance, following the injection of the BDA into the oculomotor nucleus, 
and some of these cells (all located ipsilaterally) were also positive for GABA. Based on these findings, the possibility remains that the nucleus of the medial longitudinal fasciculus and its surrounding area in the goldfish could contribute to eye-body movements, similar to those nuclei located rostral to the oculomotor nucleus in mammals.

The second major projection of GABAergic neurons to the oculomotor nucleus in the goldfish was from the rhombencephalic reticular formation. These neurons were mainly located in the medial reticular region and, to a lesser extent, in the inferior reticular region. In functional terms, reticular neurons surrounding the rostral and caudal abducens nucleus exhibit phasic-tonic and phasic signals related to horizontal eye movements (Gestrin and Sterling, 1977). Caudal to the abducens nucleus, the activity of neurons has been correlated with eye-velocity and eyeposition, and from a physiological perspective (for the lack of an explicit compelling nomenclature) these neurons are located in the hindbrain nuclei called Area II and Area I, respectively (Pastor et al., 1994; Aksay et al., 2000). On the basis of vestibular synaptic inputs, firing patterns, and embryological evidence, the Area I and Area II have been proposed as functionally analogous (not homologous) to the mammalian prepositus hypoglossi nucleus; but eye-position and eye-velocity related neurons are located into two separate hindbrain nuclei in goldfish (for further details see Straka et al., 2006). In summary, the rhombencephalic reticular formation neurons of goldfish discharge with similar patterns to the mammalian neurons involved in the circuitry for the generation of horizontal saccades (Scudder et al., 2002; Sparks, 2002). Assuming that the retrogradely labeled neurons found in the present study were saccade-related cells, it could be proposed that premotor neurons lying in the reticular formation of the goldfish are involved in the control of eye movements and fixation through a direct connection, in part GABAergic, with the oculomotor nucleus. Consistent with this proposal, monosynaptic inhibitory inputs to the oculomotor nucleus motoneurons from pontine reticular regions have been reported in mammals (Grantyn et al., 1980), and inhibitory effects from the prepositus hypoglossi nucleus upon these motoneurons have been proposed (LopezBarneo et al., 1981). This pathway could act concurrently with the abducens internuclear neurons to perform conjugate horizontal movements in vertebrates.

\section{REFERENCES}

Aksay, E., Baker, R., Seung, H.S., and Tank, D. W. (2000). Anatomy and discharge properties of pre-motor neurons in the goldfish medulla that have eyeposition signals during fixations. $J$. Neurophysiol. 84, 1035-1049.

Allum, J.H. J., Greef, N. G., and Tokunaga, A. (1981). "Projections to the rostral and caudal abducens nuclei in the goldfish," in Progress in Oculomotor Research, eds A. F. Fuchs and W. Becker (New York: Elsevier), 253-262.

Cabrera, B., Torres, B., Pasaro, R., Pastor, A. M., and Delgado-Garcia, J. M. (1992). A morphological study of abducens nucleus motoneurons and internuclear neurons in the goldfish
(Carassius auratus). Brain Res. Bull. 28, 137-144.

de la Cruz, R. R., Escudero, M., and Delgado-Garcia,J.M.(1989). Behaviour of medial rectus motoneurons in the alert cat. Eur. J. Neurol. 1, 288-295.

de la Cruz, R. R., Pastor, A. M., MartinezGuijarro, F. J., Lopez-Garcia, C., and Delgado-Garcia, J. M. (1992). Role of GABA in the extraocular motor nuclei of the cat: a postembedding immunocytochemical study. Neuroscience 51, 911-929.

Delgado-Garcia, J. M., Pozo, F. D., and Baker, R. (1986a). Behavior of neurons in the abducens nucleus of the alert cat. I. Motoneurons. Neuroscience 17, 929-952.

The major projection to the oculomotor nucleus in the goldfish was located in the nuclei of the octaval column. Neurons were found in the ipsilateral anterior octaval nucleus and in the contralateral anterior, tangential, and descending octaval nuclei. This rostro-caudal arrangement of retrogradely labeled neurons in the octaval column is similar to that found in the mammalian vestibular nuclei projection to the oculomotor nucleus, with labeled cells being reported in the ipsilateral superior vestibular nucleus and the contralateral medial, lateral, and descending vestibular nuclei (Highstein and McCrea, 1988). In addition, the most abundant source of GABAergic inputs to the oculomotor nucleus in the goldfish arises from the ipsilateral anterior octaval nucleus, with most of the neurons within the contralateral octaval nuclei being non-reactive for GABA. This pattern is consistent with the available information in the goldfish showing that electrical stimulation of the ipsilateral vestibular nerve evokes inhibitory postsynaptic potentials in the oculomotor motoneurons, whereas the stimulation of the contralateral nerve evokes excitatory postsynaptic potentials (Graf et al., 1997). Studies in frogs also support a push-pull organization of signals reaching vertical and oblique motoneurons in the oculomotor and trochlear nuclei from the second order vestibular neurons. Such organization involves an ipsilateral-GABAergic inhibitory input to the motoneurons (Straka and Dieringer, 2004). The results in fish and frogs resemble those reported in mammals, as a result of which it has been proposed that vestibular signals from each of the six canals form nearly exclusive contralateral/excitatory and ipsilateral/inhibitory three neuron arcs terminating in the appropriate extraocular motoneuron subgroups (Evinger, 1988; Highstein and McCrea, 1988). Therefore, the pattern of connectivity and GABAergic inputs to the oculomotor nucleus reported here supports the proposal by Graf et al. (1997) that homologous structural traits have been essentially preserved in the vestibulooculomotor system throughout vertebrate phylogeny.

\section{ACKNOWLEDGMENTS}

The authors wish to thank María Luisa Alvarez Parra for her technical assistance with immunohistochemistry and to Dr. Juan Luis Ribas-Salgueiro for his assistance in the microscopy service of the Seville University (CITIUS). This work was supported by the Spanish Ministerio de Innovacion y Ciencia (Grant BFU 2009-07867) and by Andalusian government grants (Proyecto de Investigacion de Excelencia: P08-CVI-039 and P09-CVI-4617).

Delgado-Garcia, J. M., Pozo, F. D., and Baker, R. (1986b). Behavior of neurons in the abducens nucleus of the alert cat. II. Internuclear neurons. Neuroscience 17, 953-973.

Easter, S. S. (1971). Spontaneous eye movements in restrained goldfish. Vision Res. 11, 333-342.

Evinger, C. (1988). "Extraocular motor nuclei: location, morphology and afferents," in Neuroanatomy of the Oculomotor System, ed. J. A. BüttnerEnnever (New York: Elsevier), 81-117.

Finger, T. E. (1978). Efferent neurons of the teleost cerebellum. Brain Res. 153 , 608-614.

Fuchs, A. F., Scudder, C. A., and Kaneko, C. R. S. (1988). Discharge patterns and recruitment order of identified motoneurons and internuclear neurons in the monkey abducens nucleus. J. Neurophysiol. 60, 1874-1895.

Gestrin, P., and Sterling, P. (1977). Anatomy and physiology of goldfish oculomotor system. II. Firing pattern of neurons in abducens nucleus and surrounding medulla and their relation to eye movements. J. Neurophysiol. 40, 573-588.

Graf, W., and McGurk, J. F. (1985). Peripheral and central oculomotor organization in the goldfish, Carassius auratus. J. Comp. Neurol. 239, 391-401.

Graf, W., Spencer, R., Baker, H., and Baker, R. (1997). Excitatory and 
inhibitory vestibular pathways to the extraocular motor nuclei in goldfish. J. Neurophysiol. 77, 2765-2779.

Grantyn, A., Grantyn, R., Gaunitz, U., and Robiné K. P. (1980). Sources of direct excitatory and inhibitory inputs from the medial rhombencephalic tegmentum to lateral and medial rectus motoneurons in the cat. Exp. Brain Res. 39, 49-61.

Highstein, S. M. (1973). Synaptic linkage in the vestibulo-ocular and trochlear reflex pathways in the rabbit. Exp. Brain Res. 17, 301-314.

Highstein, S. M., and Holstein, G. R. (2006). "The anatomy of the vestibular nuclei," in Neuroanatomy of the Oculomotor System, ed. J. A. Büttner-Ennever (New York: Elsevier), 157-203.

Highstein, S. M., and McCrea, R.A. (1988). "The anatomy of the vestibular nuclei," in Neuroanatomy of the Oculomotor System, ed. J. A. Büttner-Ennever (New York: Elsevier), 177-202.

Horn, A. K., Helmchen, C., and Wahle, P. (2003). GABAergic neurons in the rostral mesencephalon of the macaque monkey that control vertical eye movements. Ann. N. Y. Acad. Sci. 1004, 19-28.

Horn, A. K. E. (2006). "The reticular formation," in Neuroanatomy of the Oculomotor System, ed. J. A. BüttnerEnnever (New York: Elsevier), 127-155.

Ikenaga, T., Yoshida, M., and Uematsu, K. (2005). Morphology and immunohistochemistry of efferent neurons of the goldfish corpus cerebelli. J. Comp. Neurol. 487, 300-311.

Ito, M., Highstein, S. M., and Tsuchiya, T. (1970). The postsynaptic inhibition of rabbit oculomotor neurones by secondary vestibular impulses and its blockage by picrotoxin. Brain Res. 17, 520-523.

Keng, M. J., and Anastasio, T. J. (1997). The horizontal optokinetic response of the goldfish. Brain Behav. Evol. 49, 214-229.

Kobayashi, N., Yoshida, M., Matsumoto, N., and Uematsu, K. (2009). Artificial control of swimming in goldfish by brain stimulation: confirmation of the midbrain nuclei as the swimming center. Neurosci. Lett. 452, 42-46.

Lopez-Barneo, J., Ribas, J., and DelgadoGarcia, J. M. (1981). Identification of prepositus neurons projecting to the oculomotor nucleus in the alert cat. Brain Res. 214, 174-179.

Luque, M.A., Pérez-Pérez, M. P., Herrero, L., Waitzman, D. M., and Torres, B. (2006). Eye movements evoked by electrical microstimulation of the mesencephalic reticular formation in goldfish. Neuroscience 137, 1051-1073.

McCormick, C. A., and Bradford, M. R. (1994). Organization of inner-ear endorgan projection in the goldfish, Carassius auratus. Brain Behav. Evol. 43, 189-205.

Meek, J., and Nieuwenhuys, R. (1998). "Holosteans and teleosts," in The Central Nervous System of Vertebrates, eds R. Nieuwenhuys, H. J. Ten Donkelaar, and C. Nicholson (Berlin: Springer), 759-937.

Mensh, B. D., Aksay, E., Lee, D. D., Seung, H. S., and Tank, D. W. (2004). Spontaneous eye movements in goldfish: oculomotor integrator performance, plasticity, and dependence on visual feedback. Vision Res. 44, 711-726.

Nguyen, L. T., and Spencer, R. F. (1999). Abducens internuclear and ascending tract of deiters inputs to medial rectus motoneurons in the cat oculomotor nucleus: neurotransmitters. J. Comp. Neurol. 411, 73-86.

Pastor, A. M., de la Cruz, R. R., and Baker, R. (1992). Characterization and adaptive modification of the goldfish vestibuloocular reflex by sinusoidal and velocity step vestibular stimulation. J. Neurophysiol. 68 , 2003-2015.

Pastor, A. M., de la Cruz, R. R., and Baker, R. (1994). Eye position and eye velocity integrators reside in separate brainstem nuclei. Proc. Natl. Acad. Sci. U.S.A. 91, 807-811.

Pastor, A. M., de la Cruz, R. R., and Baker R. (1997). Characterization of Purkinje cells in the goldfish cerebellum during eye movement and adaptive modification of the vestibulo-ocular reflex. Prog. Brain Res. 114, 359-381.

Precht, W., Baker, R., and Okada, Y. (1973). Evidence for GABA as the synaptic transmitter of the inhibitory vestibulo-ocular pathway. Exp. Brain Res. 18, 415-428.

Scudder, C. A., Kaneko, C. S., and Fuchs, A. F. (2002). The brainstem burst generator for saccadic eye movements: a modern synthesis. Exp. Brain Res. 142, 439-462.

Sparks, D. L. (2002). The brainstem control of saccadic eye movements. Nat. Rev. Neurosci. 3, 952-964.

Spencer, R. F., and Wang, S. F. (1996) Immunohistochemical localization of neurotransmitters utilized by neurons in the rostral interstitial nucleus of the medial longitudinal fasciculus (riMLF) that project to the oculomotor and trochlear nuclei in the cat. $J$. Comp. Neurol. 366, 134-148.

Spencer, R. F., Wang, S. F., and Baker, R. (1992). The pathways and functions of GABA in the oculomotor system. Prog. Brain Res. 90, 307-331.

Straka, H., Beck, J. C., Pastor, A. M., and Baker, R. (2006). Morphology and physiology of the cerebellar vestibulolateral lobe pathways linked to oculomotor function in the goldfish. J. Neurophysiol. 96, 1963-1980.

Straka, H., and Dieringer, N. (2004). Basic organization principles of the VOR: lessons from frogs. Prog. Neurobiol. 73, 259-309.

Torres, B., Fernandez, S., Rodriguez, F., and Salas, C. (1995). Distribution of neurons projecting to the trochlear nucleus in goldfish (Carassius auratus). Brain Behav. Evol. 45, 272-285.

Torres, B., Pastor, A. M., Cabrera, B., Salas, C., and Delgado-García, J. M. (1992). Afferents to the oculomotor nucleus in the goldfish (Carassius auratus) as revealed by retrograde labeling with horseradish peroxidase. J. Comp. Neurol. 324, 449-461.

Uchino, Y., and Suzuki, S. (1983). Axon collaterals to the extraocular motoneuron pools of inhibitory vestibuloocular neurons activated from the anterior, posterior and horizontal semicircular canals in the cat. Neurosci. Lett. 37, 129-135.

Wentzel, P. R., Gerrits, N. M., and de Zeeuw, C. I. (1996). GABAergic and glycinergic inputs to the rabbit oculomotor nucleus with special emphasis on the medial rectus subdivision. Brain Res. 707, 314-319.

Conflict of Interest Statement: The authors declare that the research was conducted in the absence of any commercial or financial relationships that could be construed as a potential conflict of interest.

Received: 20 October 2010; paper pending published: 06 December 2010; accepted: 23 January 2011; published online: 04 February 2011.

Citation: Luque MA, Torres-Torrelo J, Carrascal L, Torres B and Herrero L (2011) GABAergic projections to the oculomotor nucleus in the goldfish (Carassius auratus). Front. Neuroanat. 5:7. doi: 10.3389/ fnana.2011.00007

Copyright (c) 2011 Luque, Torres-Torrelo, Carrascal, Torres and Herrero. This is an open-access article subject to an exclusive license agreement between the authors and Frontiers Media SA, which permits unrestricted use, distribution, and reproduction in any medium, provided the original authors and source are credited. 